\title{
Integrated RNAi Screening Identifies The \\ NEDDylation Pathway As A Synergistic Partner of Azacytidine In Acute Myeloid Leukemia
}

Justine Klosner ( $\square$ justine.klosner@ukmuenster.de )

University Hospital Münster

Konstantin Agelopoulos

University Hospital Münster

Christian Rohde

University Hospital Heidelberg

Stefanie Göllner

University Hospital Heidelberg

Christoph Schliemann

University Hospital Münster

Wolfgang E. Berdel

University Hospital Münster

Carsten Müller-Tidow

University Hospital Heidelberg

\section{Research Article}

Keywords: Acute Myeloid Leukemia, antileukemic therapies, synergistic, neddylation

Posted Date: July 8th, 2021

DOl: https://doi.org/10.21203/rs.3.rs-647282/v1

License: (c) (1) This work is licensed under a Creative Commons Attribution 4.0 International License.

Read Full License

Version of Record: A version of this preprint was published at Scientific Reports on December 1st, 2021. See the published version at https://doi.org/10.1038/s41598-021-02695-0. 


\section{Abstract}

Treatment of Acute Myeloid Leukemia (AML) remains challenging and novel targets and synergistic therapies still need to be discovered. We performed a high-throughput RNAi screen in three different AML cell lines and primary human leukemic blasts to identify genes that synergize with common antileukemic therapies. We used a pooled shRNA library that covered 5043 different genes and combined transfection with exposure to either azacytidine or cytarabine analog to the concept of synthetic lethality. Suppression of the chemokine CXCL12 ranked highly among the candidates of the cytarabine group. azacytidine in combination with suppression of genes within the neddylation pathway led to synergistic results. NEDD8 and RBX1 inhibition by the small molecule inhibitor pevonedistat inhibited leukemia cell growth. These findings establish an in vitro synergism between NEDD8 inhibition and azacytidine in AML. Azacytidine and NEDD8 inhibitors are currently undergoing clinical trials in combination with azacytidine. Taken together, neddylation constitutes a suitable target pathway for azacytidine combination strategies.

\section{Introduction}

Acute myeloid leukemia (AML) is the most common type of acute leukemia in adults.

Although there has been extensive research and a deeper understanding of the pathophysiology and the heterogeneous genetic background of $\mathrm{AML}$, the standard therapeutic approach for patients considered suitable candidates for intensive induction chemotherapy containing cytarabine and an anthracycline has not substantially changed for more than 40 years (Lichtman 2013; Döhner et al. 2016; Schlenk et al. 2008; Patel et al. 2012; Network 2013). Despite the intensity, therapy fails in many patients. For patients medically unfit for intensive chemotherapy low-dose cytarabine or hypomethylating agents (HMAs) 5azacytidine and decitabine are standard of care. However, these low-intensity regimens yield low response rates with even poorer median overall survival compared to intensive therapy (Dombret et al. 2015; Cashen et al. 2009).

Since refractory disease and relapse continuously are a frequent obstacle in treating patients with $A M L$, new targets to overcome chemotherapy resistance are needed.

Recently, the therapeutic landscape is rapidly changing and new drugs were approved by the Food and Drug Administration including targeted therapies that demonstrate the synergistic potential of adding targeted therapies to standard compounds (Stone et al. 2017; DiNardo et al. 2019; DiNardo et al. 2020).

RNA interference (RNAi) screens with pooled shRNA libraries are a reliable strategy for identifying new therapeutic targets (Zuber et al. 2011; Zender et al. 2008; Miller et al. 2013). Aside from the identification of potential therapeutic targets, RNAi screens can also be performed as chemosensitization screens. By exploiting the concept of synthetic lethality, new drug-combinations with synergistic effects can be identified when combining an RNAi screen with standard chemotherapy (Whitehurst et al. 2007; Giroux, lovanna, and Dagorn 2006; Porter et al. 2012; O'Neil, Bailey, and Hieter 2017). 
Aiming to identify new therapeutic targets whose downregulation sensitizes AML cells towards the two standard therapeutic agents cytarabine and azacytidine, we performed a synthetic lethal RNAi screen. To this end we used the ready-to-use barcoded Decipher shRNA library module 1 which contains 27,500 different shRNAS targeting 5,049 different genes. Since in vitro shRNA screens are often hampered by the potential problem that results could not be generalized, we conducted our RNAi screen in three different, genetically heterogeneous AML cell lines as well as in primary human leukemic cells. Statistical analysis revealed a proportionally small overlap in potential targets. Among our findings was NEDD8 to act synergistically with azacytidine. In 2009, MLN4929, a small molecule inhibitor of the NEDD8 activating enzyme (NAE) was first described (Soucy et al. 2009). Meanwhile, there are ongoing clinical investigations suggesting that the combination of pevonedistat (MNL4929) and azacytidine might act in a synthetically lethal way (Swords et al. 2018a; Swords et al. 2015a; Sekeres et al. 2021). A hypothesis that could be confirmed in this study. The exact mechanism remains to be elucidated, but we could show that this synergism seems to be specific for azacytidine.

\section{Results}

RNAi screen design and analysis

In order to identify genes that modify the response of AML cells towards the two nucleotide analogues, cytarabine and azacytidine, we performed a large-scale RNAi screen. Since AML is a genetically extremely heterogeneous disease, we screened three different AML cell lines as well as primary leukemic blasts. For our high-throughput screening approach, we chose the Decipher shRNA library module 1, which encompasses 27,500 shRNAs, expressed form a lentiviral backbone, targeting 5,049 different genes with on average five shRNAs per gene. Among the targeted genes are members of relevant signaling pathways, known drug targets and 2,500 highly ranked genes from the Cancer Genome Atlas. The shRNA library was transduced in one pool and single-cell representation was reached by limiting the multiplicity of infection rate.

Transduced cells were enriched by FACS sorting and exposed to either $100 \mathrm{nM}$ azacytidine or $100 \mathrm{nM}$ cytarabine for 72 hours and cultured for further 48 hours without treatment. Drug levels were chosen to yield $\mathrm{IC}_{20}$ values as determined in initial experiments (Supplemental Fig. 1). Cells were harvested at day 0 and day 5 . Each screen was repeated three times for each cell line resulting in nine independent experiments. AML patient blasts were harvested from one patient with refractory $A M L$, cells were analyzed at day 0 after transduction and after day 5 with and without exposure to cytarabine. For all specimens, shRNA representation was analyzed by parallel sequencing of PCR-amplified shRNA barcodes from extracted genomic DNA (Fig. 1). Next-generation sequencing was carried out with a mean coverage of 250 reads per shRNA and 8 million reads per sample. Results were normalized to the median read count of day 0 . For further analysis, data of all three cell lines were combined to identify candidates with universal synergistic results. Overall, 10 independent experiments were performed leading to a total of 412,500 data points. Due to the complex data matrix we decided to identify meaningful candidate genes based on approaches that were commonly used to analyze microarray data. Read counts for each 
shRNA and for each experiment were combined and uploaded into BRB ArrayTools, an R-based software with Excel-front end. Candidates were determined by class comparison in BRB ArrayTools, hits were further filtered by $p$-value $(P<0.05)$ and depletion rate (fold change $<0.6)$. Genes for which at least two independent shRNAs were depleted were counted as potential targets.

RNAi screen results

The screen targeted more than 5,000 genes, among them were several positive controls and 21 shRNAs targeting Luciferase as a negative control. Our approach allowed us to filter for genes that increased the cells' sensitivity towards cytarabine and azacytidine.

For the cells exposed to cytarabine, we identified 65 candidates that were significantly depleted with a negative fold change of $<0.6$. CXCL12 was identified as an enhancer of cytarabine activity (Fig. 4A). This is in line with previous reports and suggests that valid results could be obtained by our screening approach (Teicher and Fricker 2010; Chen et al. 2013).

Analysis of the data of the azacytidine group yielded 77 candidates, which included three genes involved in the proteasome cell compartment, namely NEDD8, RBX1 and PSMD11. Most significant depletion was achieved by the shRNAs targeting NEDD8 with a log-fold change of -0.82 (Fig. 3A); shRNAs targeting NEDD8 were depleted in all cell lines.

Aside from the overall analysis we also examined the results of each cell line independently and compared them with each other (Fig. 2A-C). As expected, results for each cell line differed from each other. Nonetheless, comparison of the cell lines with each other in the azacytidine-exposed arm revealed 77 mutually depleted genes, including our top candidates NEDD8, RBX1 and PSMD11 from prior analysis (Fig. 3A). Results of individual analysis for cytarabine exposed cells included the positive control CXCL12. CXCL12 was one out of 65 mutually depleted genes in AML cell lines (Fig. 4A). Matching results of cell lines and primary leukemic blasts produced 45 mutual candidates, which again included our positive control CXCL12 (Fig. 4B).

Taken together our large-scale screening approach revealed an overlap of shared potential targets, including positive controls, within all three cell lines.

Suppression of the neddylation pathway synergizes with azacytidine in sublethal concentrations

Three proteasome pathway genes were identified in our screen with two of those (NEDD8 and RBX1) both being members of the neddylation pathway. NEDD8 ranked among the top candidates on the list. In addition, NEDD8 provides a rationale target since a small molecule inhibitor (MLN4924/pevonedistat) is already used in clinical trials (Swords et al. 2015b; Swords et al. 2018b). To validate the potential candidates, we first analyzed the knockdown efficiency of the strongest depleted shRNA from the screen. Aside from NEDD8 we also sought to check for the knockdown efficiency of other shRNAs for quality control of the screening results. First, knockdown efficiency was confirmed by quantitative real-time PCR and western blot (Fig. 5A-B). Next, we sought to confirm the synergistic effect of combining NEDD8 
knockdown with exposure to azacytidine. Suppression of NEDD8 and RBX1 in HL-60 cells notably sensitized cells towards azacytidine, whereas azacytidine alone failed to inhibit the proliferation rate of HL-60 cells transfected with the negative control shRNA targeting Luciferase (Fig. 5C-D).

Next, we examined whether we could confirm these findings with pevonedistat (PEV), a small-molecule inhibitor of the NEDD8 activating enzyme (NAE). Proliferation of AML cell lines was synergistically decreased by the combination of pevonedistat and azacytidine. (Fig. 5E-F, 5H). In HL-60 cells, the combination of $100 \mathrm{nM}$ azacytidine with $25 \mathrm{nM}$ pevonedistat was equally effective as the combinations with $500 \mathrm{nM}$ azacytidine (Fig. 5E-F). Of note, the efficacy of the combination was also most pronounced in HL60 cells in the initial screen.

To exclude that diminished proliferation rates are only due to unspecific cytotoxic effects of drug addition, we also combined pevonedistat with cytarabine (Fig. 5G and I). The combination of pevonedistat and cytarabine did not lead to similar results and the addition of cytarabine to pevonedistat exhibited no synergistic effect in our experiments. The combination of azacytidine and pevonedistat was also efficient to inhibit subsequent colony formation in methylcellulose assays whereas the combination of pevonedistat and cytarabine failed to mimic this effect (Fig. $5 \mathrm{~J}-\mathrm{L}$ ). Collectively, these data suggest that the inhibition of the neddylation pathway might specifically sensitize AML cells towards sublethal concentrations of azacytidine.

\section{Discussion}

Despite recent advances in the treatment of acute myeloid leukemia, efficacy of therapy remains an important problem. To identify new potential therapeutic combinations, we conducted a large-scale RNAi screen. Comparison of the cell lines revealed only a small amount of overlapping potential targets (Fig. 2, Fig. 3, Fig. 4), thus screening several cell lines at once appears to be a useful strategy to discover promising new therapeutic approaches. Further, our screening approach allowed us to analyze a variety of aspects, e.g., antiproliferative targets and targets with synergistic effects in combination with either cytarabine or azacytidine.

We included primary human AML cells in our cytarabine screen. As already shown, RNAi screens in primary cells are feasible (Wermke et al. 2015). Our simultaneous screen of leukemic cell lines and primary human leukemic cells was followed by a comparison of cell lines and primary cells, which ranked CXCL12 among the top targets. The chemokine CXCL12 and its corresponding receptor CXCR4 on hematopoietic stem cells (HSCs) are key mediators of homing of HSCs to the bone marrow and are involved in the homeostasis of the HSCs pool - a mechanism exploited by leukemic cells. Elevated CXCR4 expression correlates with a poor prognosis in AML and suppression of CXCR4 can promote sensitivity to cytarabine (Chen et al. 2013; Rombouts et al. 2004; Uy et al. 2017; Zeng et al. 2009). In recent years small molecule inhibitors, peptide inhibitors, and anti-CXCR4 monoclonal antibodies have been developed and tested to target the CXCL12-CXCR4-axis yielding first promising results (Cho et al. 2015; Schürch 2018). 
Among our top candidates for azacytidine synergism were NEDD8 and RBX1 which both belong to the ubiquitin-proteasome-system. NEDD8, a ubiquitin-like protein, activates cullin-Ring ligases (CRLs), which are a large subgroup of E3 ubiquitin ligases (Fig. 6) (Sharad Kumar, Tomooka, and Noda 1992; S. Kumar, Yoshida, and Noda 1993; Kamitani et al. 1997). Aberrant ubiquitylation and neddylation have been linked to the development of cancer and specifically to AML (Swords et al. 2010). The proteasome inhibitor bortezomib is a successful example of the therapeutic effects of inhibition of the proteasome complex in malignant diseases (Kane et al. 2003; Kane et al. 2007; Hoeller, Hecker, and Dikic 2006). We recently showed that proteasome inhibition by bortezomib overcame a multi drug resistance phenotype in AML (Göllner et al. 2017). In addition to NEDD8, RBX1 is also involved in the ubiquitin-proteasome system and is the RING component of CRLs, a large subclass of E3 ubiquitin ligases. CRL activity is promoted by cullins, which function as molecular scaffolds and are the best-characterized NEDD8 substrates. Thus, NEDD8 and RBX1 play a pivotal role in the ubiquitylation process (Pan et al. 2004; Tan et al. 1999).

These findings suggest that neddylation pathway inhibition could synergize with azacytidine in AML. Of note, the combination of NEDD8 activating enzyme (NAE) inhibitor pevonedistat (MLN4929) with azacytidine was recently tested in a phase 1b study in AML patients (Swords et al. 2018a). This study demonstrated a high rate of complete remissions. It was previously also shown that azacytidine can help overcome pevonedistat resistance by antagonizing pevonedistat-induced Ribonucleotide Reductase subunit 2 (RRM2) increase (Visconte et al. 2016). However, preclinical data to support a specific synergism of pevonedistat and azacytidine is scarce. In this study we demonstrated that pevonedistat in combination with azacytidine disrupted the proliferation of AML cells. Further, our results suggest a specific synergistic mechanism, since the combination of pevonedistat with cytarabine did not reproduce the same antiproliferative effect (Fig. 5E-L). This is in contrast to prior studies that also showed a synergism between cytarabine and pevonedistat (Nawrocki et al. 2015). Both azacytidine and cytarabine are cytidine analogs and are incorporated into DNA, functioning as antimetabolites. Aside from cytotoxic effects, azacytidine also induces DNA demethylation by inhibiting DNA Methyltransferase 1 (DNMT1) after being incorporated into the DNA and nanomolar doses can reduce self-renewing and leukemiainitiating capacities without leading to direct cytotoxic effects (Santi, Norment, and Garrett 1984; Stresemann and Lyko 2008; Tsai et al. 2012). However, the exact mechanism of action of azacytidine is not completely understood. To identify synergistic effects, we utilized low doses of azacytidine beneath cytotoxic levels. It is possible that drug dosing precluded the identification of the previously described synergism between cytarabine and pevonedistat. Given the low doses employed, our data indicate some specificity for the azacytidine-pevonedistat synergism.

Taken together, our data provide evidence that targeting of the neddylation pathway synergizes with azacytidine to inhibit AML cell proliferation. Clinical studies shall further establish the clinical benefit of this combination.

\section{Methods}

Cell Culture 
Human leukemic cell lines were purchased from Deutsche Sammlung von Mikroorganismen und Zellkulturen (DSMZ, Braunschweig, Germany). HL-60 and Kasumi-1 were cultured in RPMI 1640 (SigmaAldrich, Darmstadt, Germany) supplemented with 10\% fetal bovine serum (Biochrom, Berlin, Germany), 1\% penicillin-streptomycin (PAA laboratories, Pasching, Austria) and 1\% L-Glutamine (Sigma-Aldrich). MV4-11 and HEK293T were cultured in IMDM (Invitrogen, Carlsbad, CA, USA), also supplemented with $10 \%$ fetal bovine serum, $1 \%$ penicillin-streptomycin and $1 \%$ L-Glutamine. All cell lines were regularly tested for mycoplasma contamination.

\section{Primary AML cells}

Primary cells were obtained from peripheral blood at the time of relapse with informed consent of the donor according to protocols approved by the institutional review boards (Ethik-Kommision der Ärztekammer Westfalen-Lippe und der Medizinischen Fakultät der Westfälischen Wilhelms Universität Münster, AZ 2007-390-f-S) and in accordance with the Declaration of Helsinki. Cells were grown in RPMI, supplemented with $20 \%$ fetal bovine serum and $5 \%$ giant cell tumor conditioned medium (Irvine Scientific, Santa Ana, CA, USA).

\section{Chemicals}

Cytarabine, azacytidine and pevonedistat (MLN4924) were purchased from Sigma-Aldrich. All drugs were stored at $-20^{\circ} \mathrm{C}$.

\section{Barcoded negative-selection RNAi screen}

The Decipher lentiviral shRNA library module 1 (Cellecta, Mountain View, CA, USA), covering 5,043 genes, contained 27,500 shRNAs in a lentiviral backbone with appropriate positive and negative controls. The library was designed to target each gene with 5 different shRNAs.

For production of lentiviral particles were produced by HEK293T cells. HEK293T cells were transfected with $6 \mu \mathrm{g}$ of lentiviral plasmid and ViraPower ${ }^{\mathrm{TM}}$ helper plasmids (Life Technologies, Darmstadt, Germany) using Lipofectamine 2000 (Invitrogen, Carlsbad, CA, USA). Supernatants containing viral particles were harvested after 48 hours and afterwards filtered through sterile syringe filters (Thermo Fisher Scientific, Waltham, MA, USA) and stored at $-80^{\circ} \mathrm{C}$.

For lentiviral transduction dishes were coated with RetroNectin ${ }^{\circledR}$ (Fisher Scientific, Waltham, MA, USA). To guarantee sufficient library representation total number of 30 million cells were used for transduction and at least a quantity of 20 million was maintained during the experiment. $72 \mathrm{~h}$ after transduction, infected cells were selected by cell sorting using a BD FACSAria ${ }^{\mathrm{TM}}$ III (BD Biosciences, San Jose, CA, USA) and subsequently expanded for approximately further $72 \mathrm{~h}$ until required cell number was reached. At this time point, 20 million cells were harvested (day 0 ) and frozen at $-20^{\circ} \mathrm{C}$. Remaining cells were split into three treatment groups and exposed to either $100 \mathrm{nM}$ azacytidine, $100 \mathrm{nM}$ cytarabine or to DMSO for $72 \mathrm{~h}$. After treatment cells were kept in culture for further 48 hours and harvested on day 5. Genomic DNA from samples harvested at day 0 and day 5 was extracted using the DNeasy Blood\&Tissue Kit (Qiagen, Hilden, 
Germany) according to the manufacturer's protocol. DNA was further concentrated by ethanol precipitation. Next, two rounds of PCR for barcode amplification followed as described elsewhere (Fredebohm et al. 2013). Before sequencing analysis, samples were marked with a specific barcode and multiplexed with Encore TM 384 Multiplex System (NuGen Technologies, Leek, NL). A total of 12pmol gDNA was used. Decipher barcode abundance was analyzed by next-generation sequencing with a HiScanTMSQ System (Illumina, San Diego, USA).

\section{Quantitive Real-Time PCR (qPCR)}

To analyze expression of mRNA HEK293T cells were transduced with highest scoring shRNA for PSMD11, NEDD8 and RBX1. RNA was isolated using RNeasy Mini Kit (Qiagen, Hilden, Germany). Quantitive PCR was performed according to manufacturer's instructions with SYBR green. All signals were normalized to levels of Gapdh and results were calculated by the $\Delta \mathrm{Ct}$ method.

Western blot analyisis

Positively transduced cells were lysed using the radioimmunoprecipitation assay (RIPA) buffer. SDSPAGE and western blotting were performed as already described (Tickenbrock et al. 2006). Antibodies against NEDD8 and RBX1 were obtained from Cell Signaling Technology (Danvers, MA, USA), an antibody against PSMD11 was obtained from Novus Biologicals (Littleton, CO, USA).

\section{Proliferation Assays}

Cell profliferation assays were performed by cell counting or by MTS assays. All assays were done in triplicate. MTS assays (Promega Corp., Fitvhburg, WI USA) were used for the determination of $\mathrm{IC}_{50}$. HL-60, Kasumi-1 and MV4-11 were treated with azacytidine or cytarabine from $5 \mathrm{~nm}$ to $1000 \mathrm{~nm}$. Cell proliferation was measured after 48 hours with a Benchmark microplate reader (Bio-Rad, Hercules, CA, USA).

In vitro shRNA Proliferation assays were performed with HL-60, Kasumi-1, and MV4-11. Transduced cells were selected as described above and exposed to $100 \mathrm{nM}$ azacytidine for 72 hours. For inhibitor testing, cells were exposed to $25 \mathrm{nM}$ pevonedistat in combination with either $100 \mathrm{nM}, 500 \mathrm{nM}$ azacytidine or $25 \mathrm{nM}$ cytarabine for 72 hours. Subsequently, cells were transferred to fresh medium. Viable cells were counted at day 5 using the TC20TM Automated Cell Counter (Bio-Rad, Hercules, CA USA), dead cells were excluded by Trypan Blue staining. Relative proliferation rates were calculated by normalizing to mocktreated cells.

Methylcellulose Colony-Forming Assay

HL-60 cells were exposed to pevonedistat in combination with azacytidine or cytarabine in a 3-day drug treatment scheme; medium was exchanged every 24 hours. An equal number of cells (500 per plate) were plated in triplicates on to methylcellulose M0512 (Sigma-Aldrich) supplemented with fetal bovine serum and L-Glutamine. Colonies containing more than 40 cells were scored after 7 days. 


\section{Statistical analysis}

Raw data of sequencing analysis was first exported using the software Illumina CASAVA Software Version 1.8.2. Next sequences were analyzed by the "Barcode Deconvoluter" software provided by Cellecta. Further analysis was performed in BRB ArrayTools, an R-based software with Excel-front end.

Statistical differences were analyzed by Student's t-test using GraphPad Prism software. A p-value $<0.05$ was defined as a significant difference.

\section{Declarations}

\section{Acknowledgments}

The authors thank Beate Lindtner for excellent technical assistance and Stephanie Lettermann for assistance with NGS library preparation.

\section{References}

1. Cashen, A. F., Gary, J., Schiller, Margaret, R., O’Donnell \& John F. DiPersio Multicenter, Phase II Study of Decitabine for the First-Line Treatment of Older Patients With Acute Myeloid Leukemia. Journal of Clinical Oncology, 28 (4), 556-561 https://doi.org/10.1200/jco.2009.23.9178 (2009).

2. Chen, Y. et al. CXCR4 Downregulation of Let-7a Drives Chemoresistance in Acute Myeloid Leukemia. Journal of Clinical Investigation, 123 (6), 2395-2407 https://doi.org/10.1172/jci66553 (2013).

3. Cho, B. S. et al. Antileukemia Activity of the Novel Peptidic CXCR4 Antagonist LY2510924 as Monotherapy and in Combination with Chemotherapy., 126 (2), 222-232 https://doi.org/10.1182/blood-2015-02-628677 (2015).

4. DiNardo, C. D. et al. Azacitidine and Venetoclax in Previously Untreated Acute Myeloid Leukemia. New England Journal of Medicine, 383 (7), 617-629 https://doi.org/10.1056/nejmoa2012971 (2020).

5. DiNardo, C. D. et al. Venetoclax Combined with Decitabine or Azacitidine in Treatment-Naive, Elderly Patients with Acute Myeloid Leukemia., 133 (1), 7-17 https://doi.org/10.1182/blood-2018-08868752 (2019).

6. Döhner, H. et al. Diagnosis and Management of AML in Adults: 2017 ELN Recommendations from an International Expert Panel., 129 (4), 424-447 https://doi.org/10.1182/blood-2016-08-733196 (2016).

7. Dombret, Hervé, John F. Seymour, Aleksandra Butrym, Agnieszka Wierzbowska, Dominik Selleslag, Jun Ho Jang, Rajat Kumar, et al. 2015. "International Phase 3 Study of Azacitidine vs Conventional Care Regimens in Older Patients with Newly Diagnosed AML with $>30 \%$ Blasts." Blood 126 (3): $291-$ 299. doi:10.1182/blood-2015-01-621664.

8. Fredebohm, J., Wolf, J., Hoheisel, J. Ã. D. \& Michael Boettcher Depletion of RAD17 Sensitizes Pancreatic Cancer Cells to Gemcitabine. Journal of Cell Science, 126 (15), 3380-3389 
https://doi.org/10.1242/jcs.124768 (2013).

9. Giroux, V. \& Juan lovanna, and Jean-Charles Dagorn Probing the Human Kinome for Kinases Involved in Pancreatic Cancer Cell Survival and Gemcitabine Resistance. The FASEB Journal, 20 (12), 1982-1991 https://doi.org/10.1096/fj.06-6239com (2006).

10. Göllner, S. et al. Loss of the Histone Methyltransferase EZH2 Induces Resistance to Multiple Drugs in Acute Myeloid Leukemia. Nature Medicine, 23 (1), 69-78 https://doi.org/10.1038/nm.4247 (2017).

11. Hoeller, D., Hecker, C. M. \& Dikic, I. Ubiquitin and Ubiquitin-like Proteins in Cancer Pathogenesis. Nature Reviews Cancer, 6 (10), 776-788 https://doi.org/10.1038/nrc1994 (2006).

12. Kamitani, T., Kito, K., Nguyen, H. P., Edward, T. H. \& Yeh Characterization of NEDD8, a Developmentally Down-Regulated Ubiquitin-like Protein. Journal of Biological Chemistry, 272 (45), 28557-28562 https://doi.org/10.1074/jbc.272.45.28557 (1997).

13. Kane, R. C., Peter, F., Bross, Ann, T., Farrell \& Richard Pazdur Velcade ${ }^{\circledR}:$ U.S. FDA Approval for the Treatment of Multiple Myeloma Progressing on Prior Therapy., 8 (6), 508-513 https://doi.org/10.1634/theoncologist.8-6-508 (2003).

14. Kane, R. C. et al. Bortezomib for the Treatment of Mantle Cell Lymphoma. Clin. Cancer Res, 13 (18), 5291-5294 https://doi.org/10.1158/1078-0432.ccr-07-0871 (2007).

15. Kumar, S., Yoshida, Y. \& Noda, M. Cloning of a CDNA Which Encodes a Novel Ubiquitin-like Protein. Biochemical and Biophysical Research Communications, 195 (1), 393-399 https://doi.org/10.1006/bbrc.1993.2056 (1993).

16. Kumar, S., Tomooka, Y. \& Makoto Noda Identification of a Set of Genes with Developmentally DownRegulated Expression in the Mouse Brain. Biochemical and Biophysical Research Communications, 185 (3), 1155-1161 https://doi.org/10.1016/0006-291x(92)91747-e (1992).

17. Lichtman, M. A. A Historical Perspective on the Development of the Cytarabine (7days) and Daunorubicin (3days) Treatment Regimen for Acute Myelogenous Leukemia: 2013 the 40th Anniversary of $7+3$. Blood Cells, Molecules, and Diseases, 50 (2), 119-130 https://doi.org/10.1016/j.bcmd.2012.10.005 (2013).

18. Miller, P. G. et al. In Vivo RNAi Screening Identifies a Leukemia-Specific Dependence on Integrin Beta 3 Signaling., 24 (1), 45-58 https://doi.org/10.1016/j.ccr.2013.05.004 (2013).

19. Nawrocki, S. T. et al. The NEDD8-Activating Enzyme Inhibitor MLN4924 Disrupts Nucleotide Metabolism and Augments the Efficacy of Cytarabine. Clin. Cancer Res, 21 (2), 439-447 https://doi.org/10.1158/1078-0432.ccr-14-1960 (2015).

20. Network \& The Cancer Genome Atlas Research. Genomic and Epigenomic Landscapes of Adult De Novo Acute Myeloid Leukemia. The New England Journal of Medicine, 368 (22), 2059-2074 https://doi.org/10.1056/nejmoa1301689 (2013).

21. O’Neil, N. J., Bailey, M. L. \& Philip Hieter Synthetic Lethality and Cancer. Nature Reviews Genetics, 18 (10), 613-623 https://doi.org/10.1038/nrg.2017.47 (2017).

22. Pan, Z. Q., Kentsis, A., Dias, D. C., Yamoah, K. \& Kenneth Wu Nedd8 on Cullin: Building an Expressway to Protein Destruction., 23 (11), 1985-1997 https://doi.org/10.1038/sj.onc.1207414 (2004). 
23. Patel, J. P. et al. Prognostic Relevance of Integrated Genetic Profiling in Acute Myeloid Leukemia. The New England Journal of Medicine, 366 (12), 1079-1089 https://doi.org/10.1056/nejmoa1112304 (2012).

24. Porter, C. C. et al. Integrated Genomic Analyses Identify WEE1 as a Critical Mediator of Cell Fate and a Novel Therapeutic Target in Acute Myeloid Leukemia., 26 (6), 1266-1276 https://doi.org/10.1038/leu.2011.392 (2012).

25. Rombouts, E. J. C., Pavic, B., Löwenberg, B. \& Rob E. Ploemacher Relation between CXCR-4 Expression, Flt3 Mutations, and Unfavorable Prognosis of Adult Acute Myeloid Leukemia., 104 (2), 550-557 https://doi.org/10.1182/blood-2004-02-0566 (2004).

26. Santi, D. V., Norment, A. \& Garrett, C. E. 1984. "Covalent Bond Formation between a DNA-Cytosine Methyltransferase and DNA Containing 5-Azacytosine." Proceedings of the National Academy of Sciences 81 (22): 6993-6997. doi:10.1073/pnas.81.22.6993.

27. Schlenk, R. F. et al. Mutations and Treatment Outcome in Cytogenetically Normal Acute Myeloid Leukemia. The New England Journal of Medicine, 358 (18), 1909-1918 https://doi.org/10.1056/nejmoa074306 (2008).

28. Schürch, C. M. Therapeutic Antibodies for Myeloid Neoplasms-Current Developments and Future Directions. Frontiers in Oncology, 8, 152 https://doi.org/10.3389/fonc.2018.00152 (2018).

29. Sekeres, M. A. et al. Randomized Phase 2 Trial of Pevonedistat plus Azacitidine versus Azacitidine for Higher-Risk MDS/CMML or Low-Blast AML., 1-6 https://doi.org/10.1038/s41375-021-01125-4 (2021).

30. Soucy, T. A. et al. An Inhibitor of NEDD8-Activating Enzyme as a New Approach to Treat Cancer. Nature, 458 (7239), 732-736 https://doi.org/10.1038/nature07884 (2009).

31. Stone, R. M. et al. Midostaurin plus Chemotherapy for Acute Myeloid Leukemia with a FLT3 Mutation. The New England Journal of Medicine, 377 (5), 454-464 https://doi.org/10.1056/nejmoa1614359 (2017).

32. Stresemann, C. \& Frank Lyko Modes of Action of the DNA Methyltransferase Inhibitors Azacytidine and Decitabine. International Journal of Cancer, 123 (1), 8-13 https://doi.org/10.1002/ijc.23607 (2008).

33. Swords, R. T. et al. Pevonedistat, a First-in-Class NEDD8-Activating Enzyme Inhibitor, Combined with Azacitidine in Patients with AML., 131 (13), 1415-1424 https://doi.org/10.1182/blood-2017-09805895 (2018a).

34. Swords, R. T. et al. Pevonedistat, a First-in-Class NEDD8-Activating Enzyme Inhibitor, Combined with Azacitidine in Patients with AML., 131 (13), 1415-1424 https://doi.org/10.1182/blood-2017-09805895 (2018b).

35. Swords, R. T. et al. Pevonedistat (MLN4924), a First-in-Class NEDD8-activating Enzyme Inhibitor, in Patients with Acute Myeloid Leukaemia and Myelodysplastic Syndromes: A Phase 1 Study. British Journal of Haematology, 169 (4), 534-543 https://doi.org/10.1111/bjh.13323 (2015a). 
36. Swords, R. T. et al. Pevonedistat (MLN4924), a First-in-Class NEDD8-activating Enzyme Inhibitor, in Patients with Acute Myeloid Leukaemia and Myelodysplastic Syndromes: A Phase 1 Study. British Journal of Haematology, 169 (4), 534-543 https://doi.org/10.1111/bjh.13323 (2015b).

37. Swords, R. T. et al. Inhibition of NEDD8-Activating Enzyme: A Novel Approach for the Treatment of Acute Myeloid Leukemia., 115 (18), 3796-3800 https://doi.org/10.1182/blood-2009-11-254862 (2010).

38. Tan, P. et al. Ze'ev Ronai, and 1999. "Recruitment of a ROC1-CUL1 Ubiquitin Ligase by Skp1 and HOS to Catalyze the Ubiquitination of IKBa." Molecular Cel/3 (4): 527-533. doi:10.1016/s10972765(00)80481-5.

39. Teicher, B. A. \& Fricker, S. P. CXCL12 (SDF-1)/CXCR4 Pathway in Cancer. Clin. Cancer Res, 16 (11), 2927-2931 https://doi.org/10.1158/1078-0432.ccr-09-2329 (2010).

40. Tsai, H. C. et al. Transient Low Doses of DNA-Demethylating Agents Exert Durable Antitumor Effects on Hematological and Epithelial Tumor Cells., 21 (3), 430-446 https://doi.org/10.1016/j.ccr.2011.12.029 (2012).

41. Uy, G. L. et al. A Phase 1/2 Study of Chemosensitization with Plerixafor plus G-CSF in Relapsed or Refractory Acute Myeloid Leukemia. Blood Cancer Journal, 7 (3), e542-e542 https://doi.org/10.1038/bcj.2017.21 (2017).

42. Visconte, V. et al. Comprehensive Quantitative Proteomic Profiling of the Pharmacodynamic Changes Induced by MLN4924 in Acute Myeloid Leukemia Cells Establishes Rationale for Its Combination with Azacitidine., 30 (5), 1190-1194 https://doi.org/10.1038/leu.2015.250 (2016).

43. Wermke, M. et al. RNAi Profiling of Primary Human AML Cells Identifies ROCK1 as a Therapeutic Target and Nominates Fasudil as an Antileukemic Drug., 125 (24), 3760-3768 https://doi.org/10.1182/blood-2014-07-590646 (2015).

44. Whitehurst, A. W. et al. Synthetic Lethal Screen Identification of Chemosensitizer Loci in Cancer Cells. Nature, 446 (7137), 815-819 https://doi.org/10.1038/nature05697 (2007).

45. Zender, L. et al. An Oncogenomics-Based In Vivo RNAi Screen Identifies Tumor Suppressors in Liver Cancer., 135 (5), 852-864 https://doi.org/10.1016/j.cell.2008.09.061 (2008).

46. Zeng, Z. et al. Targeting the Leukemia Microenvironment by CXCR4 Inhibition Overcomes Resistance to Kinase Inhibitors and Chemotherapy in AML., 113 (24), 6215-6224 https://doi.org/10.1182/blood2008-05-158311 (2009).

47. Zuber, J. et al. RNAi Screen Identifies Brd4 as a Therapeutic Target in Acute Myeloid Leukaemia. Nature, 478 (7370), 524-528 https://doi.org/10.1038/nature10334 (2011).

\section{Figures}


Colonies of HL-60, Kasumi-1, and MV4-11

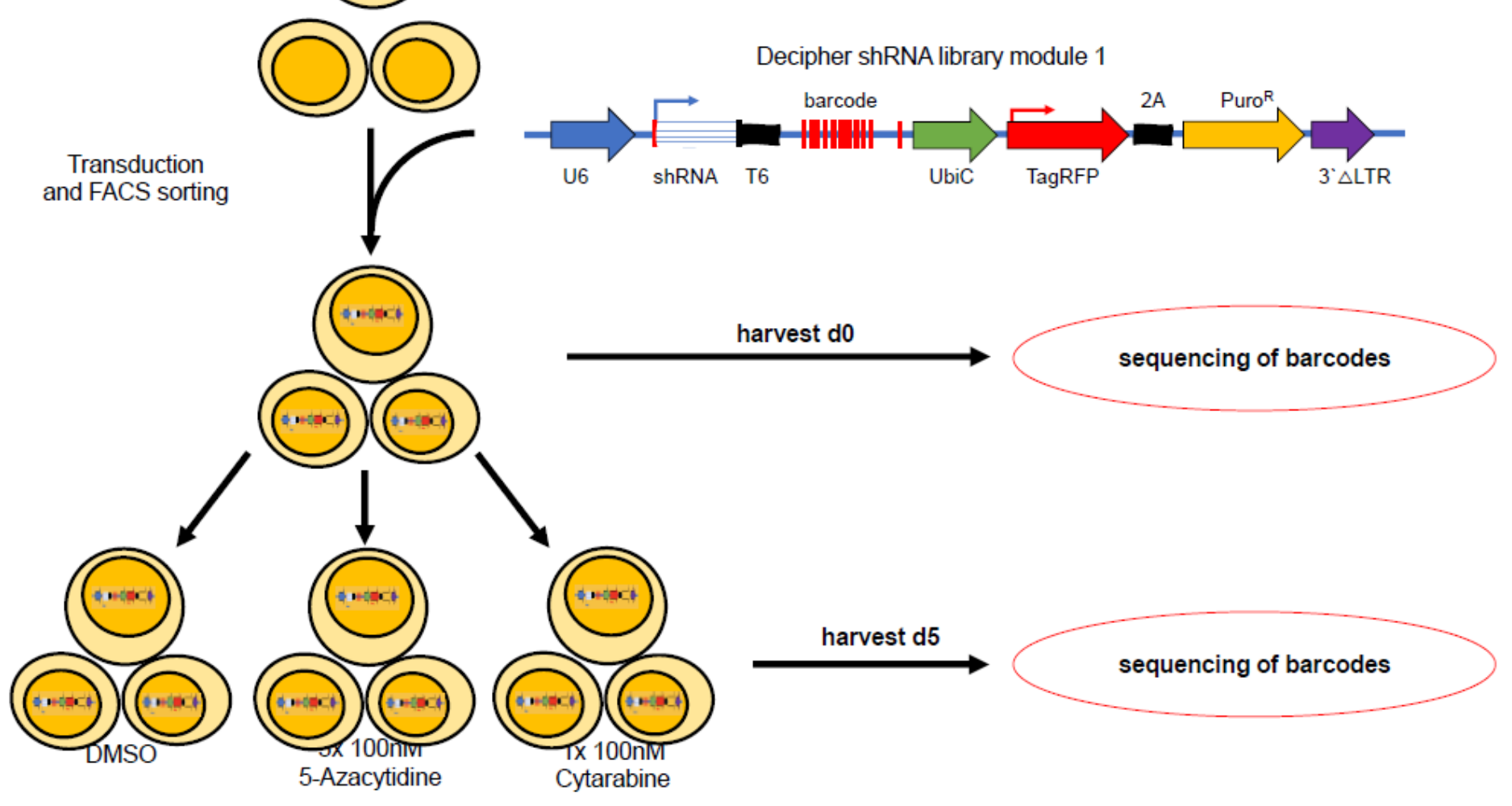

Figure 1

Pooled RNAi screens in leukemia cell lines and primary blasts. (A) The in vitro shRNA screen was performed in three different cell lines and in primary leukemia blasts, resulting in ten independent experiments. Successfully transduced cells were selected via FACS sorting for TagRFP, expanded and subsequently exposed to either cytarabine, azacytidine or DMSO for 72 hours. Cells were harvested at day 0 and day 5 , genomic DNA was extracted and shRNA abundance was analyzed by next generation sequencing. 
A

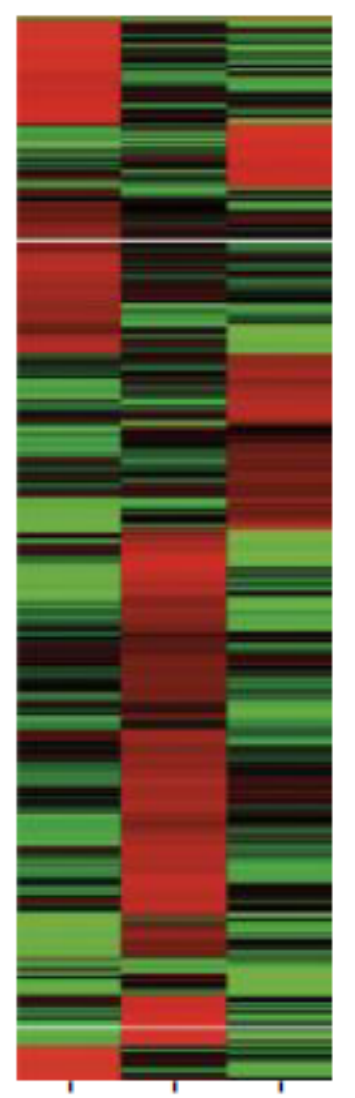

$\mathrm{B}$

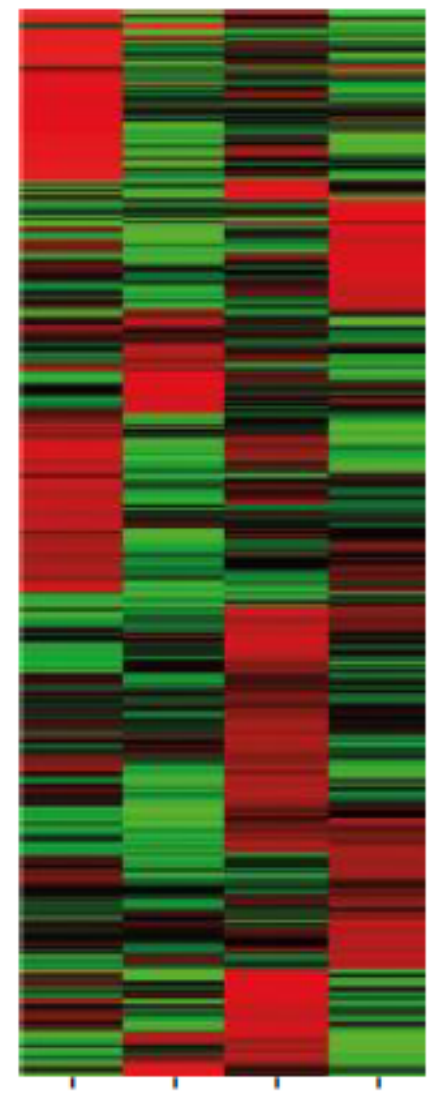

$\mathrm{C}$

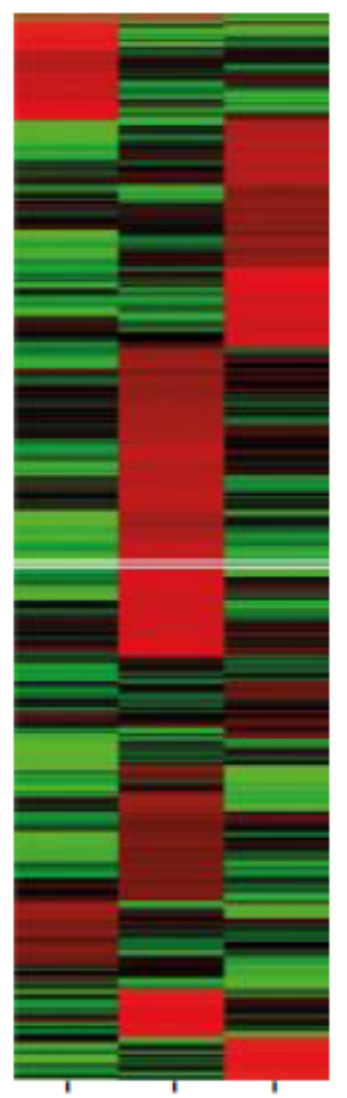

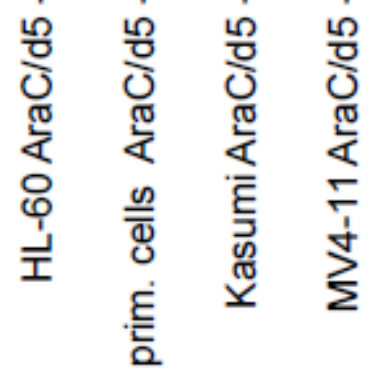

\section{Figure 2}

Screening heterogenous cell lines leads to only few potential targets. (A) Heatmap showing the alterations of representation of 27,500 shRNAs in HL-60, Kasumi-1 and MV4-11 exposed to sublethal concentrations of azacytidine (AZA) at day 5. (B) Heatmap showing the alterations of representation of 27,500 shRNAs in HL-60, Kasumi-1, MV4-11 and primary human blasts exposed to sublethal concentrations of cytarabine (AraC) at day 5. (C) Heatmap depicting changes in representation of 27,500 shRNAs over the course of 5 days in HL-60, Kasumi-1 and MV4-11. 
A

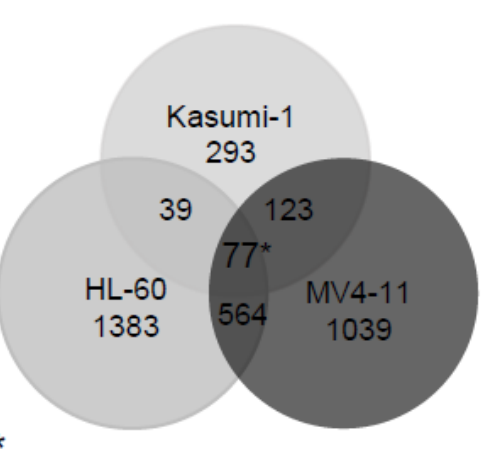

$\star$

\begin{tabular}{|ll|}
\hline ABCB5 & PDE4C \\
ALDOC & PFKFB3 \\
ANAPC2 & POLR2B \\
AP2M1 & PRKAB2 \\
BID & PROC \\
C7ORF16 & PRPF19 \\
CAMK2G & PSMA3 \\
CAV3 & PSMB1 \\
CIP29 & PSMD11 \\
COL16A1 & PSMD2 \\
COX15 & PTPRO \\
CP & RAN \\
DFFB & RBX1 \\
EFTUD2 & RPA2 \\
EIF2S1 & RPL10 \\
ENG & RPL11 \\
FBL & RPL13A \\
FCGR3A & RPL14 \\
GFPT1 & RPS6 \\
HNRNPC & RPSA \\
HOXA1 & RRM1 \\
IGFBP3 & RTN4 \\
IL17F & RUVBL1 \\
IL28RA & SAP18 \\
KCNMB2 & SCTR \\
KIF11 & SEC22B \\
KPNB1 & SF1 \\
LCK & SIN3A \\
LOC388720 & SLPI \\
MEF2D & SMN1 \\
MMAB & SMPD1 \\
MTHFD1L & SNAP23 \\
MUT & SNAP47 \\
MYB & SNRPA1 \\
NAGS & TAF13 \\
NEDD8 & TBL1XR1 \\
NQO1 13 TOP1 \\
NUP214 & TUBA1C \\
P2RY12 & \\
\hline
\end{tabular}

B

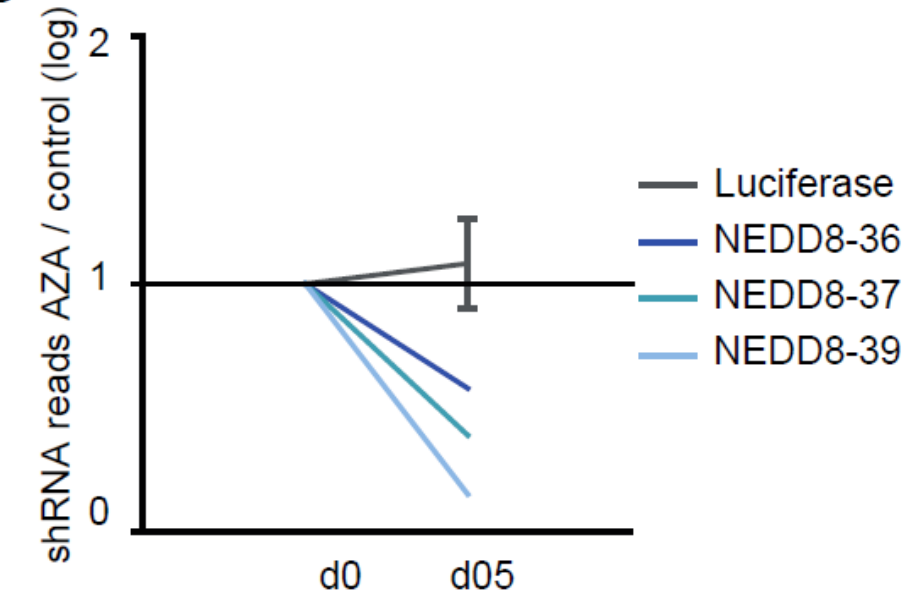

C

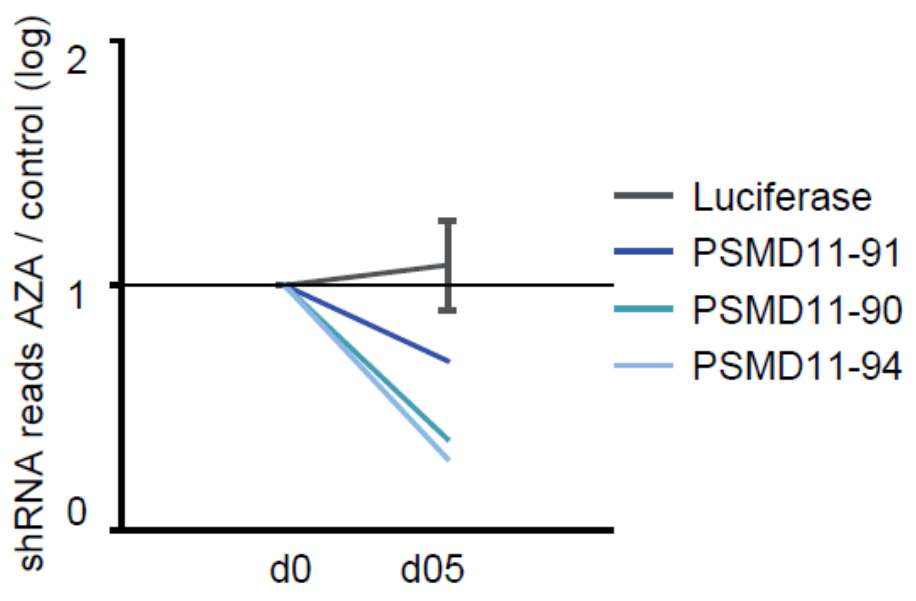

D

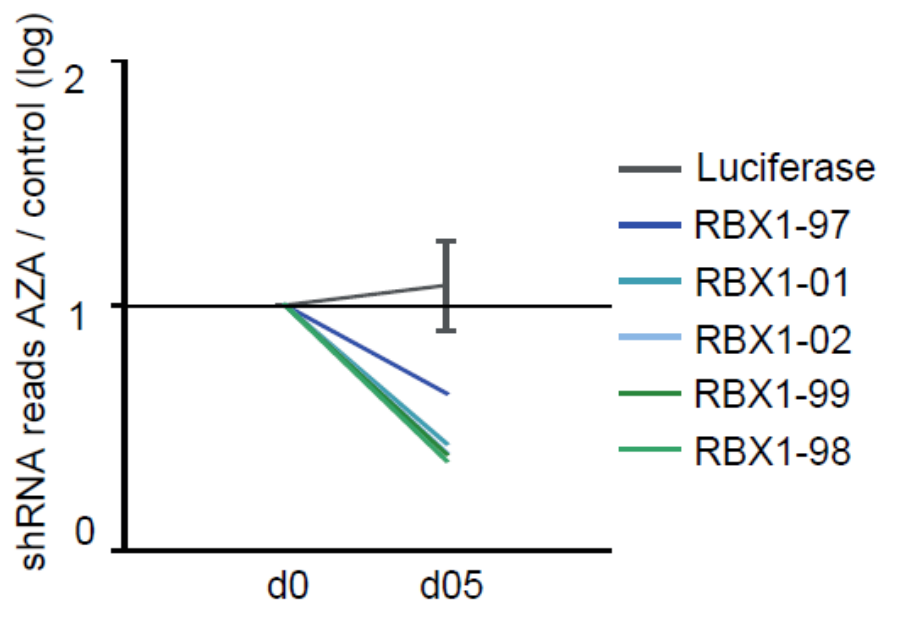

\section{Figure 3}

Comparison of results in the Azacytidine group. (A) Venn diagram shows the number of potential candidates in each tested cell line in the AZA/d05 analysis and visualizes mutually depleted genes (fold change $<0.6)$ with at least two independent shRNAs targeting the same gene. Among them are NEDD8, RBX1 and PSMD11, all part of the proteasome complex. (B) - (D) Plots showing median depletion of different shRNAs targeting NEDD8 (B), PSMD-11 (C) and RBX-1(D) in HL-60, Kasumi-1 and MV4-11. 
A

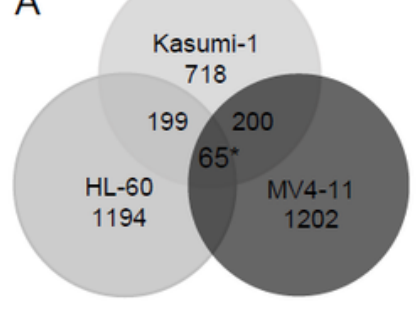

\begin{tabular}{|ll|}
\hline ACTR2 & KLK10 \\
AMDHD1 & LSS \\
APOA5 & MYB \\
ATP4A & MYLK2 \\
ATP5C1 & NPC1 \\
BID & NRG1 \\
CAD & PAFAH2 \\
CASP12 & PIGK \\
CD247 & PIK3R3 \\
CHD8 & PINK1 \\
CLEC5A & PLK1 \\
CXCL12 & POLR2A \\
DAXX & POLR2B \\
DBT & POLR2D \\
DCLRE1A & PRKCD \\
DDX56 & PRPF40A \\
DNAJA2 & PSMA3 \\
DNAJB9 & RAN \\
DVL2 & RBX1 \\
EBP & RPL10 \\
EIF2S1 & RPS13 \\
ESD & SFRS12IP1 \\
FCGR3B & SIN3A \\
FOLR4 & SLC6A11 \\
GABRB3 & SORBS1 \\
GATA1 & SRY \\
GGA3 & SSRP1 \\
GOT2 & SYNE1 \\
HTR2C & TGDS \\
IL1RN & TSG101 \\
IMPDH1 & UQCRC2 \\
KARS & KIF11 \\
\hline
\end{tabular}

C

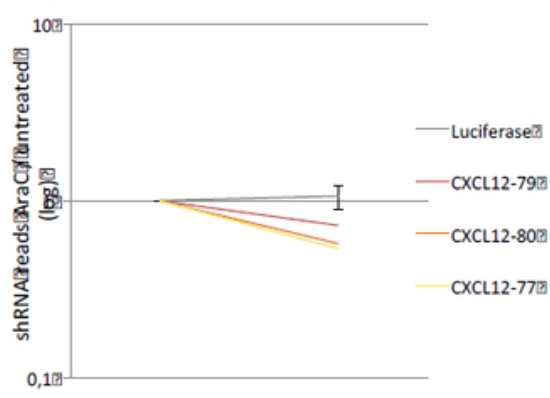

B

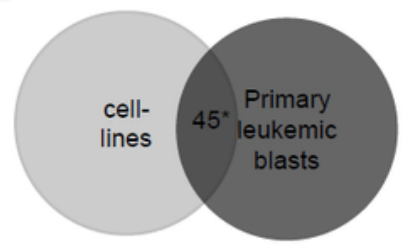

\begin{tabular}{|c|c|}
\hline AMDHD1 & KARS \\
\hline APOA5 & KIF11 \\
\hline ATP4A & MYB \\
\hline ATP5C1 & $\mathrm{NPC1}$ \\
\hline BID & PIK3R3 \\
\hline CAD & PLK1 \\
\hline CASP12 & POLR2A \\
\hline CD247 & POLR2B \\
\hline CLEC5A & POLR2D \\
\hline $\mathrm{CXCL} 12$ & PRKCD \\
\hline DAXX & PSMA3 \\
\hline DDX56 & RAN \\
\hline DNAJA2 & RBX1 \\
\hline DNAJB99 & RPL10 \\
\hline EBP & RPS13 \\
\hline EIF2S1 & SFRS12IP1 \\
\hline ESD & SIN3A \\
\hline FCGR3B & SLC6A11 \\
\hline FOLR4 & SSRP1 \\
\hline GATA1 & TGDS \\
\hline GGA3 & TSG01 \\
\hline GOT2 & XPA \\
\hline
\end{tabular}

D

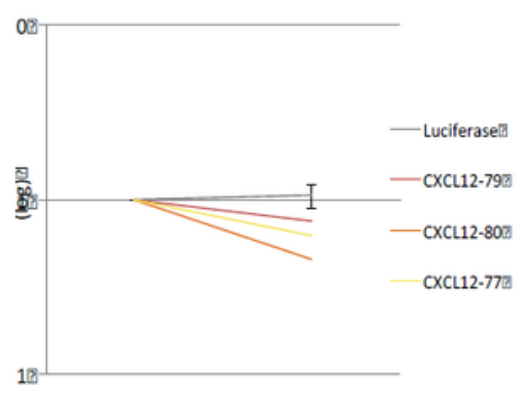

\section{Figure 4}

Comparison of results in the cytarabine group and primary cells. (A) Venn diagram shows the number of potential therapeutic targets in each tested cell line in the AraC/d05 analysis and visualizes mutually depleted genes (fold change $<0.6$ ) with at least two independent shRNAs targeting the same gene. Among the 65 hits is CXCL12. (B) Venn diagram shows mutually depleted genes of cell lines (HL-60, Kasumi-1, MV4-11) and primary leukemic blasts when comparing AarC/d05 ratio. Again, only targets with 
two independent shRNAs were taken into account. (C) - (D) Plots showing median depletion of different shRNAs targeting CXCL12 in cell lines (C) and in primary leukemic blasts (D).

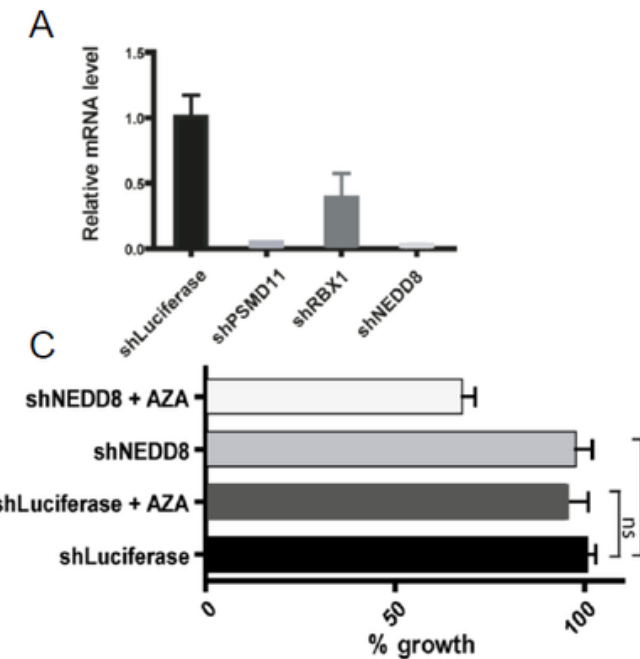

$\mathrm{E}$
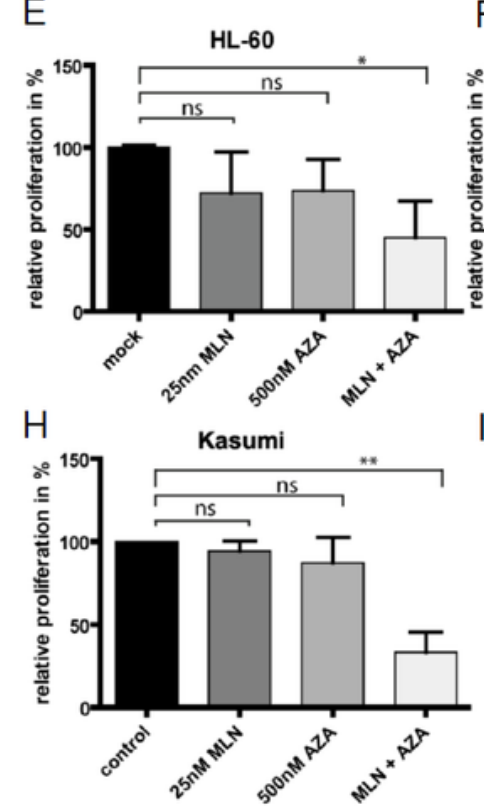

$\mathrm{F}$
B

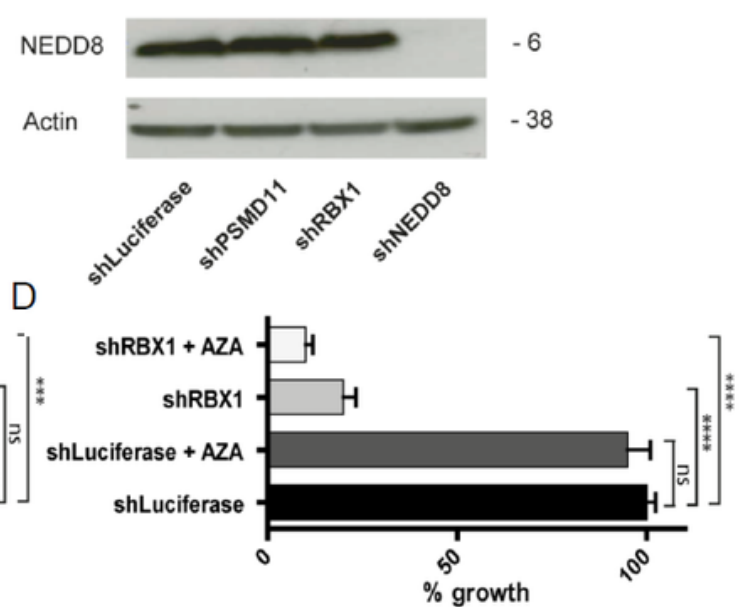

G
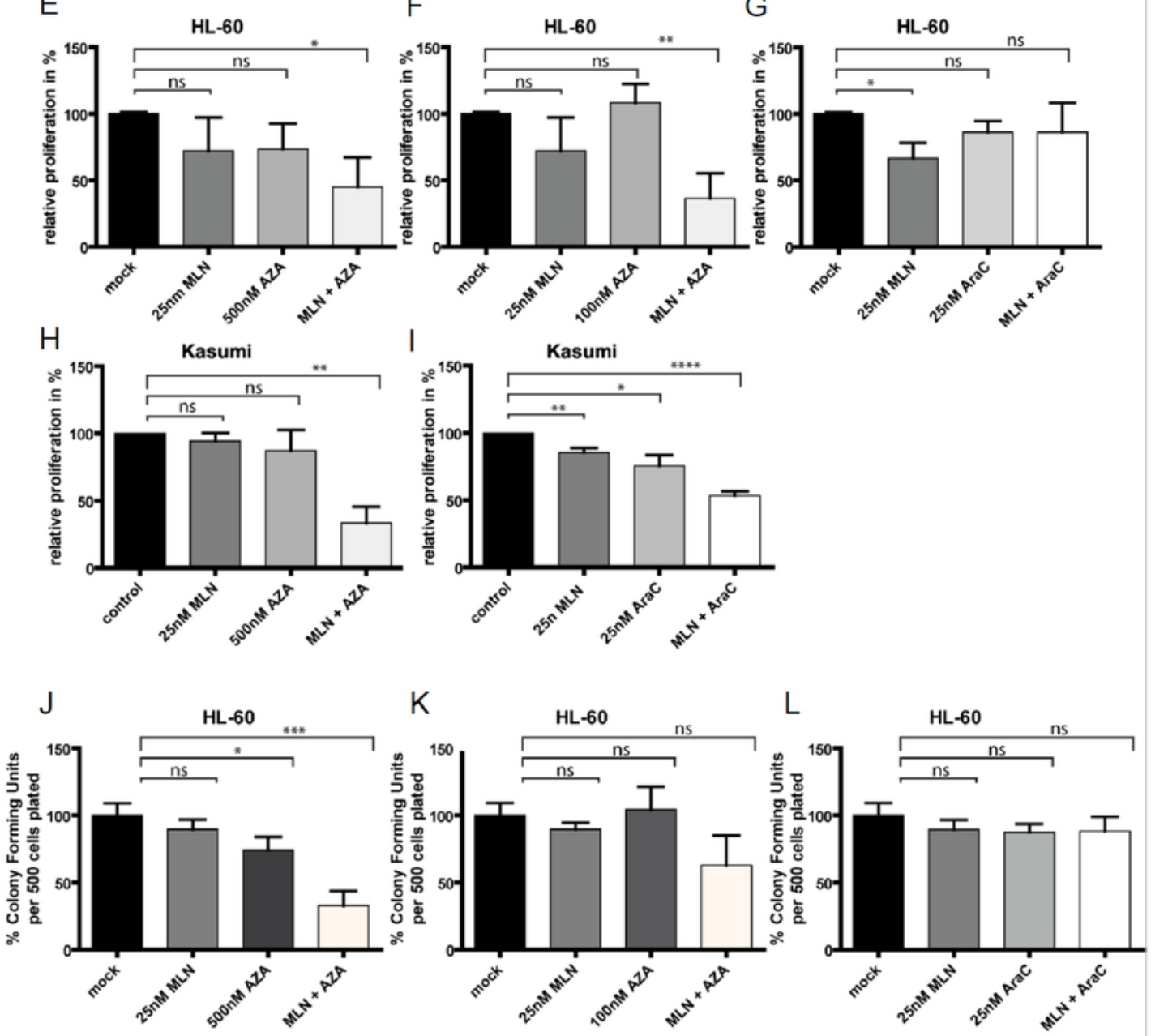

Figure 5

Validation of NEDD8 as a synergistic target in AML (A) MRNA levels of NEDD8, RBX1, and PSMD11 were assessed in HL-60 cells transfected with corresponding shRNA and control shRNA. (B) Protein levels were measured in HL-60 with Western blotting of whole-cell lysates. (C)-(D) Proliferation rates of HL-60 cells 
transfected with shRNAs targeting either NEDD8 or RBX1 and Luciferase as control. Transfected cells were exposed to $500 \mathrm{nM}$ azacytidine (AZA) or DMSO for 24 hours. Proliferation rates were calculated by cell counting after 72 hours and normalized to the proliferation rate of DMSO treated cells. $(E)-(I)$ Proliferation rate of HL-60 and Kasumi-1 exposed to NEDD8 inhibitor pevonedistat (PEV) in combination with either azacytidine (AZA) or cytarabine (AraC). Proliferation rate was measured by cell counting and calculated as explained above. $(\mathrm{J})-(\mathrm{L})$ Methylcellulose colony-forming assays were performed in $\mathrm{HL}-60$ cells after 72 hours daily treatment in vitro with either azacytidine (AZA) or cytarabine (AraC) in combination with pevonedistat (PEV). Controls were treated with DMSO.

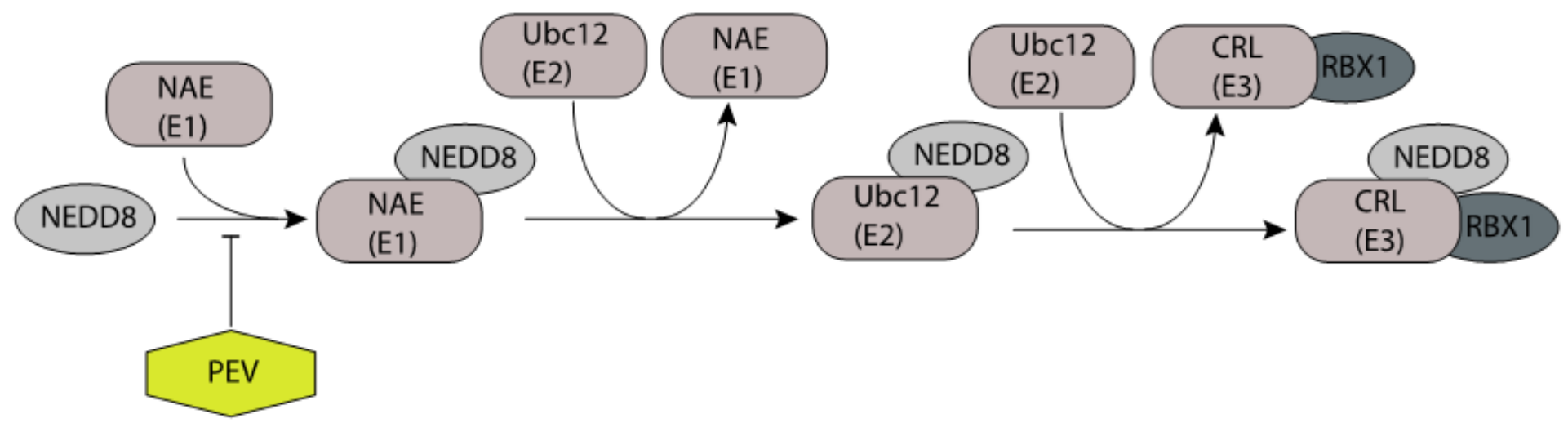

\section{Figure 6}

Neddylation pathway and its inhibitor pevonedistat The activation of the largest ubiquitin ligases subgroup cullin-ring ligases (CRLs) starts with activation of NEDD8 by the NEDD8 E1 activation enzyme (NAE) in an ATP-dependent reaction. Afterwards, it transferers to the conjugation enzyme E2. The NEDD8 charged E2 then conjugates NEDD8 to a cullin-containing RING finger ligase (CRL). The neddylation pathway can be disrupted by the small molecule inhibitor pevonedistat, which inhibits NAE.

\section{Supplementary Files}

This is a list of supplementary files associated with this preprint. Click to download.

- SupplementalFigure1.pptx

- SupplementalFigureLegend.docx 